\title{
Can a toxin gene NAAT be used to predict toxin EIA and the severity of Clostridium difficile infection?
}

\author{
Mark I. Garvey ${ }^{1,2^{*}}$ (D), Craig W. Bradley ${ }^{1}$, Martyn A. C. Wilkinson ${ }^{1}$ and Elisabeth Holden ${ }^{1}$
}

\begin{abstract}
Background: Diagnosis of $C$. difficile infection (CDI) is controversial because of the many laboratory methods available and their lack of ability to distinguish between carriage, mild or severe disease. Here we describe whether a low C. difficile toxin B nucleic acid amplification test (NAAT) cycle threshold (CT) can predict toxin EIA, CDI severity and mortality.

Methods: A three-stage algorithm was employed for CDI testing, comprising a screening test for glutamate dehydrogenase (GDH), followed by a NAAT, then a toxin enzyme immunoassay (EIA). All diarrhoeal samples positive for GDH and NAAT between 2012 and 2016 were analysed. The performance of the NAAT CT value as a classifier of toxin EIA outcome was analysed using a ROC curve; patient mortality was compared to CTs and toxin ElA via linear regression models.
\end{abstract}

Results: A CT value $\leq 26$ was associated with $\geq 72 \%$ toxin ElA positivity; applying a logistic regression model we demonstrated an association between low $C T$ values and toxin EIA positivity. A CT value of $\leq 26$ was significantly associated $(p=0.0262)$ with increased one month mortality, severe cases of CDI or failure of first line treatment. The ROC curve probabilities demonstrated a CT cut off value of 26.6.

Discussions: Here we demonstrate that a $C T \leq 26$ indicates more severe CDI and is associated with higher mortality. Samples with a low CT value are often toxin EIA positive, questioning the need for this additional ElA test.

Conclusions: $A C T \leq 26$ could be used to assess the potential for severity of CDI and guide patient treatment.

Keywords: Clostridium Difficile, NAAT, EIA, Severity, Mortality, C. difficile Infection

\section{Introduction}

C. difficile is an anaerobic, spore forming Gram positive bacillus found in diverse environments that is able to colonise and proliferate in the human gastrointestinal tract, especially following changes in the indigenous colonic microbiota after antibiotic use $[1,2]$. C. difficile is one of the most common causes of healthcare associated infection and is responsible for 15 to $25 \%$ of all cases of antibiotic associated diarrhoea [2-4]. The infection causes an estimated 3000 deaths every year in the UK and 15,000-20,000 deaths in the USA, with associated

\footnotetext{
* Correspondence: mark.garvey@uhb.nhs.uk; m.i.garvey@bham.ac.uk
${ }^{1}$ University Hospitals Birmingham NHS Foundation Trust, Queen Elizabeth

* Correspondence: mark.garvey@uhb.nhs.uk; m.i.garvey@bham.ac.uk
${ }^{1}$ University Hospitals Birmingham NHS Foundation Trust, Queen Elizabeth Hospital Birmingham, B15 2WB, Edgbaston, Birmingham, UK ${ }^{2}$ Institute of Microbiology and Infection, The University of Birmingham, Edgbaston, Birmingham, UK
}

case-fatality rates of $6-17 \%[4,5]$. It is associated with gut overgrowth of $C$. difficile and the production of toxins A, B, or both, which cause a range of effects, including gut mucosal damage, colitis, and pseudomembranous colitis $[2,4]$. The primary mediators of inflammation in C. difficile infection (CDI) are large clostridial toxins, toxin A and toxin $\mathrm{B}$. The toxins trigger a complex cascade of host cellular responses to cause diarrhoea, inflammation and tissue necrosis - the major symptoms of CDI [2, 4]. It must be noted a small number of healthy people naturally carry $C$. difficile in their large intestine and do not have ill effects from the infection [1].

Since $C$. difficile diarrhoea cannot be reliably distinguished from other causes of healthcare associated diarrhoea on clinical grounds alone, laboratory confirmation is essential [4, 6-10]. However, the diagnosis of CDI 
remains a contentious issue $[4,8,9,11]$. For decades, toxin tests were favoured over culture for diagnosis of CDI because toxins mediate disease, detection was faster and this method provided evidence of toxin production in vivo that typically correlated better with clinical disease $[3,10,12,13]$. Molecular tests such as nucleic acid amplification tests (NAAT) target toxin genes but are similar to culture in detecting $C$. difficile bacteria regardless of toxin production, making it unclear whether positive NAAT results reflect clinical disease $[4,12,14]$. A disadvantage for NAATs is that they detect toxin genes alone and not toxin production which is thought to be associated with CDI [15]. The uncertain clinical significance of positive NAAT results is problematic in inpatient healthcare facilities where $C$. difficile colonization is 5 to 10 times more common than CDI and non-infectious causes of diarrhoea [4, 12, 15]. A large multi-centre study in the UK led to a change in the Department of Health diagnostic guidance, after showing that an algorithmic approach was optimal for laboratory diagnosis [4]. Two stage algorithms have been widely adopted, and consist of; an initial screen using glutamate dehydrogenase (GDH) enzyme immunoassay (EIA) or toxin gene NAAT to detect the presence of $C$. difficile, followed by a second step which detects faecal toxin using either EIA or a cell cytotoxin assay $[4,8,11,13$, $16,17]$. The cell cytotoxin assay is used to detect the presence of $C$. difficile toxin by its effect on human tissue cells grown in culture. This test is considered to be the 'gold standard' for diagnosing CDI, however it requires technical expertise and takes 24 to $48 \mathrm{~h}$ to produce a result [10].

In the UK, screen positive/toxin negative patients are usually regarded as being colonized with $C$. difficile rather than infected, based on a large multicentre prospective study showing that only patients with detectable faecal toxin had adverse outcomes [2-4]. However, outside the UK, such patients typically identified with NAATs as a screening test alone, are often regarded as having CDI $[3,18]$. The role of $C$. difficile toxin gene NAAT for CDI diagnosis has largely been polarised between Europe and the US [3, 19]. Data from the National Healthcare Safety Network indicated in $2014 \sim 44 \%$ of US hospitals use NAAT for CDI diagnosis, whilst in Europe that level was much lower at $5 \%[3,19]$. Over diagnosis of CDI in hospitals using standalone NAAT can lead to unnecessary treatment causing clinicians to miss the real underlying diagnosis [3]. The outcome of patients who are NAAT positive/ toxin EIA negative has been shown to be indistinguishable from that of NAAT negative and toxin EIA negative patients (in terms of diarrhoea and CDI related complications) [3, 15]. Additionally, increased mortality is associated with the presence of faecal toxin but not with NAAT positive/ toxin negative sample results [15]. While qualitative results of NAAT have poor positive predictive value for $\mathrm{CDI}$, could there be an alternative role for NAATs in CDI diagnosis? [12, 15] Here we aimed to determine if a low NAAT cycle threshold result can predict severity of CDI and/or mortality using data from our institution over the past 5 years.

\section{Materials and methods \\ Setting}

Queen Elizabeth Hospital Birmingham (QEHB), part of University Hospitals Birmingham (UHB) NHS Foundation Trust is a tertiary referral National Health Service teaching hospital in Birmingham, UK that provides clinical services to over one million patients every year.

\section{C. difficile Testing}

In line with national guidance, an algorithmic approach to identifying CDI is undertaken at QEHB $[4,20,21]$. A three-stage algorithm is employed. Briefly, any patient with $\geq 1$ episode of unexplained diarrhoea had their faecal specimen tested for CDI. The CDI testing algorithm consists of an initial screening step using a Premier GDH EIA (Meridian Bioscience, Cincinnati, Ohio), followed by a NAAT (Cepheid, Xpert ${ }^{\mathrm{m}} \mathrm{C}$. difficile, US) for GDH positive samples only. The premier GDH involved undertaking an enzyme immunoassay looking for the presence of GDH as previously descrbed $[4,21]$. The Cepheid NAAT is a real-time PCR assay targeting the toxin genes ( $B$ toxin gene and $t c d B$ ) $[12,14]$. The Cepheid PCR targets the toxin $\mathrm{B}$ gene $(\mathrm{t} c d B)$ as it is independently capable of causing CDI $[12,14]$. The cycle threshold (CT) value of the Cepheid real time PCR describes the number of cycles needed until DNA amplification occurs exponentially in a real-time PCR assay and they are correlated with the amount of target sequence in the sample [12]. All samples which were GDH and NAAT positive have a Premier Toxins A and B EIA (Meridian Bioscience, Cincinnati, Ohio) which is undertaken once a week [20, 22]. The Premier Toxins A and B EIA was undertaken as previously described, with a sensitivity of $94.7 \%$ and specificity $97.3 \%$ quoted by the manufacturers when compared to the reference cytotoxin method $[4,21]$.

\section{Study design and definitions}

All diarrhoeal samples (Bristol stool type 5-7) [23] from patients between Jan 2012 to Dec 2016 at QEHB positive by GDH and NAAT were included in the study which equated to 1346 patients. 


\section{CDI episode}

At QEHB a CDI episode was defined as the presence of a positive test result for toxigenic $C$. difficile by $\mathrm{GDH}$ and NAAT and the presence of unexplained diarrhoea ( $\geq 1$ episode of unexplained diarrhoea). Severity of CDI was based on the Public Health England (PHE) toolkit for management of $C$. difficile [20]. A severe $C$. difficile case was defined as: WCC >15 $\times 10$ [9] g/dL; acutely rising blood creatinine (e.g. $>50 \%$ increase above baseline); temperature $>38.5{ }^{\circ} \mathrm{C}$; or evidence of severe colitis (abdominal signs, radiology) [20].

\section{Recurrent $\mathrm{CDI}$ and treatment failure}

Recurrent CDI was defined as the return of diarrhoea ( $\geq 1$ episode of unexplained diarrhoea) within 30 days of a previous CDI episode and the presence of a positive test result for toxigenic $C$. difficile by GDH and NAAT [20]. Treatment failure was defined as cases where failure to respond to treatment resulted in a change of management of the patient [12].

\section{Clinical data collection}

Patient data collected at the time of a positive result included: patient demographics (age, sex), markers for CDI severity (white cell count, C-reactive protein, serum creatinine, serum albumin, temperature, stool frequency) and mortality (one month and 3-month all-cause mortality). Clinical severity data were obtained from 80 patients with the lowest and 138 patients with the highest CT values; mortality data was collected from all 1346 patients in the study.

\section{Statistical analysis}

Cepheid NAAT CT values were compared to Premier Toxins A and B EIA positivity and patient mortality (one and three month). All analyses including receiver operating characteristic curve (ROC), Youden's index, error rates and univariate logistic regression models were performed using the hmeasure, plotROC and base packages in the statistical programming language R [24-26].

\section{ROC curve}

The performance of NAAT CT value as a classifier of toxin EIA positivity was assessed using a ROC curve and the area under the ROC curve (AUC). The sensitivity (TPF; True Positive Fraction), specificity, False Positive Fraction (FPF), Positive Predictive Value (PPV) and negative predictive value (NPV) of the prediction rule of low $\mathrm{CT}$ values corresponding to EIA positivity were calculated using the hmeasure and plotROC packages in $\mathrm{R}$ $[24,25]$. To determine a NAAT CT cut off value, two commonly-used strategies were used in the study picking a threshold that minimises the error rate and picking one that maximises Youden's index [27, 28].

\section{Linear regression mortality}

Logistic regression models were used to assess NAAT CT values as an explanatory variable for mortality and EIA toxin positivity. These models were fitted using $\mathrm{R}$ [26].

\section{Results \\ Samples}

Between Jan 012012 and Dec 31 2016; 26,931 diarrhoeal stool samples were tested for CDI at QEHB. Of the 26,931 samples 2807 (10\%) were GDH positive, of these 1650 (59\%) were NAAT positive. Of the $1650 \mathrm{GDH}$ and NAAT positives, 754 (46\%) were toxin EIA positive (Fig. 1).

\section{NAAT CT vs EIA}

The mean toxin B CT-value of all samples was 27.1 $(n=1650)$. Low CT values were associated with toxin EIA positivity (Table 1 ). A CT value of $<26$ was associated with $\geq 72 \%$ toxin EIA positivity as compared to a CT value $\geq 26$ where a $23 \%$ EIA positivity rate was observed (Table 1). To test the model of low CT values corresponding to EIA positivity a ROC curve was calculated (Fig. 2). The area under the ROC curve (AUC) was equal to AUC $=0.819$ (Table 2). For our data, we have a minimum error rate of

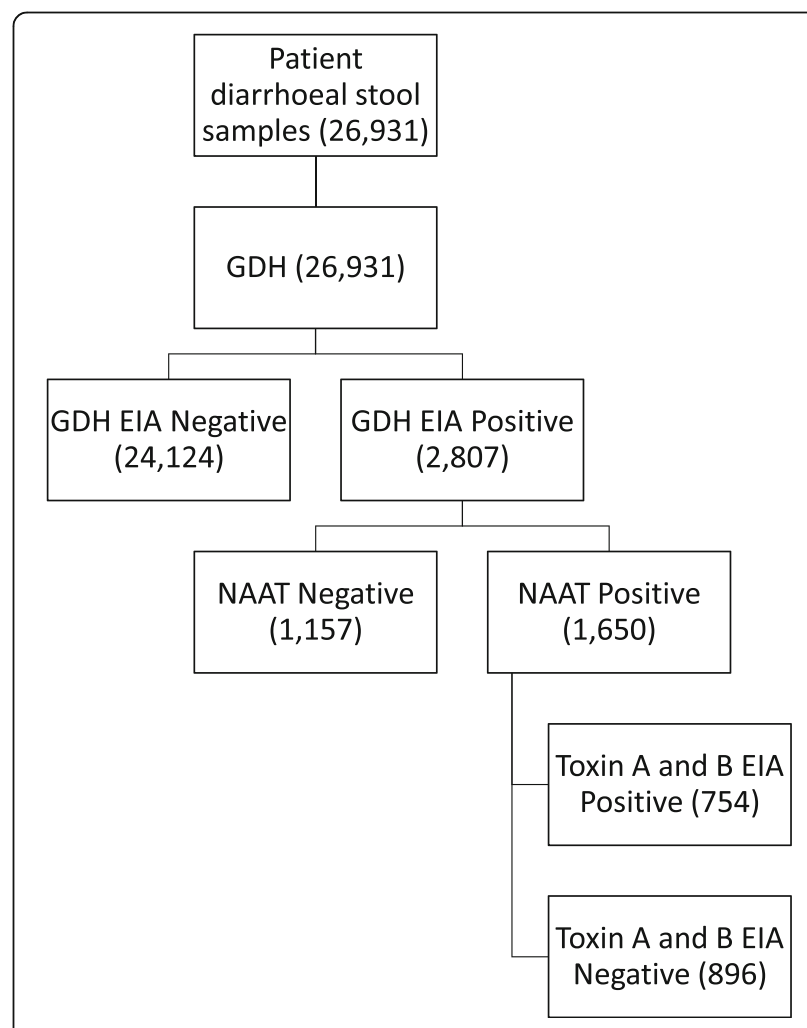

Fig. 1 Algorithm of CDI testing at QEHB with the number of results obtained between Jan 012012 and Dec 312016 depicted 
Table 1 The number of toxin EIA samples against a selection of NAAT CT value ranges with patient mortality within 1 month and 3 months

\begin{tabular}{|c|c|c|c|c|}
\hline CT Value & EIA Toxin Positive ${ }^{a}$ & Total EIA b & Mortality/month ${ }^{c}$ & Mortality/3 months ${ }^{c}$ \\
\hline 18 & $1(100 \%)$ & 1 & 1 & 1 \\
\hline 19 & $4(80 \%)$ & 5 & 2 & 2 \\
\hline 20 & 32 (84\%) & 38 & 7 & 12 \\
\hline 21 & $80(85 \%)$ & 94 & 17 & 25 \\
\hline 22 & $113(80 \%)$ & 140 & 16 & 29 \\
\hline 23 & 119 (72\%) & 166 & 23 & 31 \\
\hline 24 & $106(67 \%)$ & 157 & 27 & 39 \\
\hline 25 & $96(56 \%)$ & 171 & 21 & 34 \\
\hline 26 & $63(50 \%)$ & 125 & 12 & 19 \\
\hline 27 & $52(37 \%)$ & 139 & 19 & 34 \\
\hline 28 & $24(27 \%)$ & 89 & 7 & 13 \\
\hline 29 & $23(24 \%)$ & 95 & 10 & 16 \\
\hline 30 & $17(20 \%)$ & 83 & 8 & 16 \\
\hline $31-37$ & $24(7 \%)$ & 347 & 41 & 60 \\
\hline All & $754(46 \%)$ & 1650 & 210 & 331 \\
\hline $18-25$ & $551(72 \%)$ & 772 & 113 & 173 \\
\hline $26-37$ & $203(23 \%)$ & 878 & 97 & 158 \\
\hline
\end{tabular}

${ }^{a}$ All samples are GDH, NAAT and toxin A and B EIA positive

${ }^{b}$ Total number of toxin $A$ and $B$ EIAs undertaken from GDH and NAAT positive samples including both negative and positive results for toxin $A$ and $B$

${ }^{c}$ Reflects the number of patients with 1 month or 3-month mortality

approximately 0.251 which occurs at toxin thresholds 26.6 and 26.7, whilst the maximum value for Youden's index is approximately 0.507, which occurs at the toxin threshold 26.7. A cut off $\mathrm{CT}$ value $\leq 26$ was chosen as a value likely to yield EIA positivity, whilst minimising the risk of over diagnosis (Table 2).

To investigate whether NAAT CT value correlates with toxin EIA positivity, the following logistic regression model was used: $\mathrm{Y}=9.50654-0.36306 \mathrm{X}$ (where the response variable, $Y$, is the logit function of the probability of a positive result from the toxin EIA, and $X$ is the NAAT CT value). The $p$ value for the regression coefficient for the explanatory variable is $p$ $<2 \times 10^{-16}$, demonstrating that the CT value there is inversely proportional to the probability of a positive result from the toxin EIA. The model predicts the approximate probabilities of a positive EIA result as $0.904,0.606,0.517,0.200$ and 0.039 for the $\mathrm{CT}$ values $20,25,26,30$ and 35 , respectively.

\section{NAAT CT vs mortality}

Between 2012 and 2016, 210 patients died within a month of a $C$. difficile NAAT positive result, this increased to 331 after 3 months (Table 1); 805 patients did not die. A logistic regression model was used to identify whether CT value corresponds with mortality. When looking at the number of deaths within 1 month, the following regression model was used: $Y=-0.85203-0.03996 X$ (the response variable, $Y$, is the logit function of 1-month mortality, and the explanatory variable, $X$, is the NAAT CT value). The $p$ value for the regression coefficient for the explanatory variable is $p=0.0262$, indicating that a low CT value is associated with mortality. The model predicts the approximate probabilities of death within 1 month as $0.161,0.136,0.114$ and 0.095 for the CT values $20,25,30$ and 35 , respectively.

\section{NAAT CT vs severity of CDI}

Clinical data were obtained from 80 patients with the lowest and 138 patients with the highest CTs. 99 out of 138 (72\%) patients with a CT value between 18 and 21 had severe/recurrent CDI. Failure of first line treatment with metronidazole was observed in 23 out of the 39 cases with low CT values that had been classified as mild CDI, resulting in escalation to second line therapy such as vancomycin, fidaxomicin or faecal microbiota therapy. Of the 80 patients with CT values between 35 and 37, 74 (92\%) of the patients had mild CDI with resolution of symptoms following first line therapy of metronidazole.

\section{Discussion}

In our patient population, analysis of all $C$. difficile NAAT positive stool samples between 2012 and 2016 


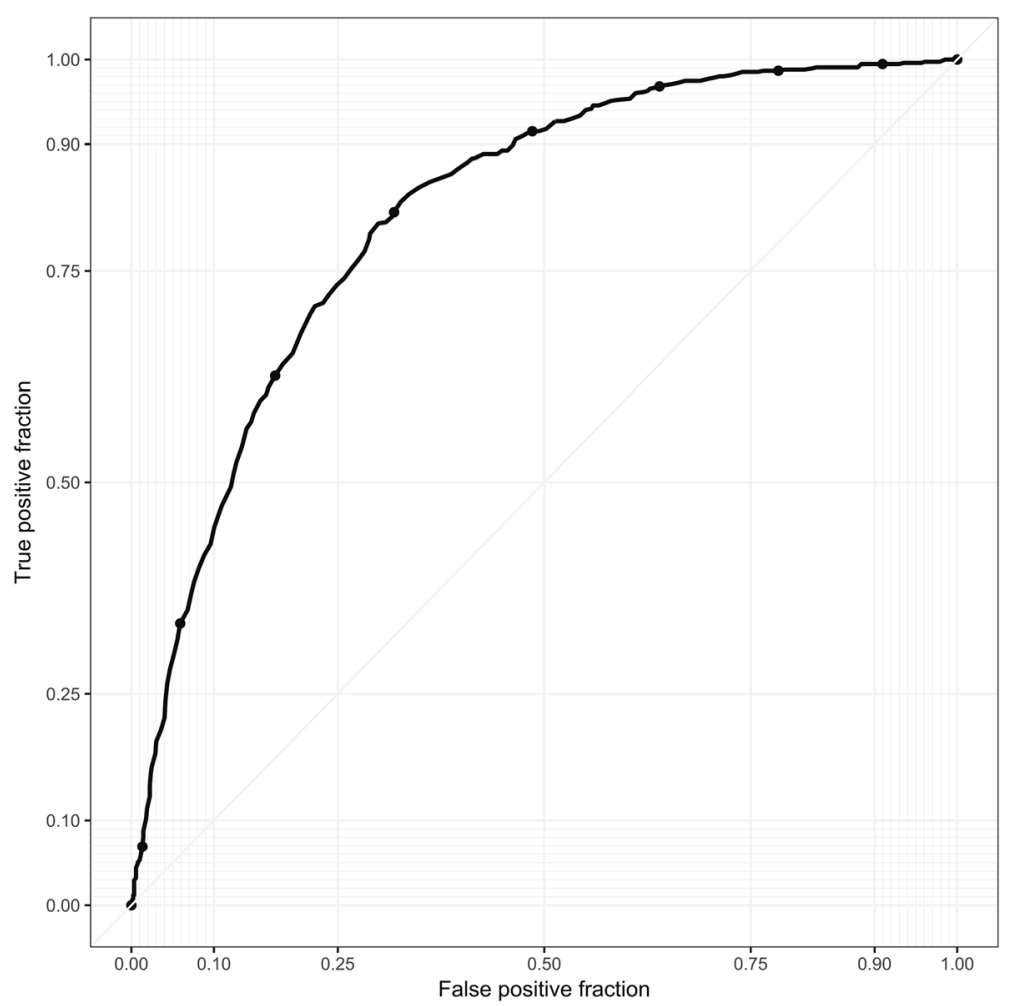

Fig. 2 ROC curve comparing NAAT CT value vs toxin EIA positivity. Note: The area under the ROC curve (AUC) was equal to AUC $=0.819$. The $y$-axis is the True Positive Fraction, or sensitivity, and the $x$-axis is the False Positive Fraction, which is equal to 1 -specificity. The True Positive Fraction is the probability that for a fixed cut off, $c$, the classifier $(M)$ gives a positive result when the true result $(D)$ is positive, i.e. $\operatorname{TPF}(c)=P(M \geq c \mid$ $D=$ Positive). In a similar fashion, the False Positive Fraction is the probability that for a fixed cut off the classifier gives a positive result when the true result is negative, i.e. $\operatorname{TPF}(c)=P(M \geq c \mid D=$ Negative $)$

Table 2 Comparison of NAAT CT values (1/C) vs toxin EIA positivity

\begin{tabular}{llllll}
\hline CT value & Sensitivity (TPF) & Specificity & FPF & PPV & NPV \\
\hline 20 & 0.008 & 0.998 & 0.002 & 0.750 & 0.544 \\
21 & 0.050 & 0.992 & 0.008 & 0.844 & 0.554 \\
22 & 0.163 & 0.975 & 0.025 & 0.848 & 0.581 \\
23 & 0.314 & 0.944 & 0.056 & 0.826 & 0.621 \\
24 & 0.472 & 0.891 & 0.109 & 0.784 & 0.667 \\
25 & 0.613 & 0.834 & 0.166 & 0.756 & 0.719 \\
26 & 0.733 & 0.751 & 0.249 & 0.713 & 0.770 \\
27 & 0.820 & 0.682 & 0.318 & 0.684 & 0.818 \\
28 & 0.883 & 0.585 & 0.415 & 0.642 & 0.856 \\
29 & 0.915 & 0.507 & 0.493 & 0.610 & 0.876 \\
30 & 0.948 & 0.426 & 0.574 & 0.582 & 0.907 \\
35 & 0.995 & 0.076 & 0.924 & 0.475 & 0.944 \\
\hline
\end{tabular}

The table shows $\mathrm{CT}$ values against various diagnostic measures used in the ROC analysis including sensitivity and specificity. The area under the ROC curve (AUC) was equal to $A \cup C=0.819$, corresponding to a $C T$ value of $\leq 26$ TPF True Positive Fraction, FPF False Positive Fraction, PPV Positive Predictive Value, NPV Negative Predictive Value indicated that a low NAAT CT value was independently associated with toxin EIA positivity, higher mortality and CDI severity. We propose that a microbiological marker cut off, based on CT value $(\leq 26)$, could be used as a predictor of poor outcome and serve as a severity indicator to guide treatment of CDI.

In this study, regression analysis demonstrated that low CT values were associated with toxin EIA positivity. With a CT value $\leq 26$ we saw $\geq 72 \%$ toxin EIA positivity; applying a logistic regression model we demonstrated an association between low CT values and toxin EIA positivity. Conversely a $\mathrm{CT}$ value of $\leq 26$ would miss $28 \%$ of patients that were EIA positive. It is not surprising that a low CT value is linked with toxin EIA positivity as previous studies have suggested expression of toxin is associated with the severity of CDI, and a correlation between faecal toxin levels and $C$. difficile counts has been reported in mice [12]. Dionne et al., previously identified that amplification CT values correlate to bacterial load [29]. They demonstrated toxin positive samples had higher bacterial loads than toxin negative samples and 
there was a significant inverse correlation between PCR CT values and bacterial loads [29]. In the present study, low CT values inversely correlate to toxin assay positivity, one could imply that CT values of toxin $\mathrm{B}$ genes act as a marker of the amount of toxin produced [12]. However, investigations seeking to link quantitative toxin production with $C$. difficile virulence have not been conclusive [12]. Further work is warranted to explore this.

In the UK, two stage algorithms for CDI have been adopted $[4,10]$. Often a GDH test for screening is employed followed by a toxin EIA $[4,10]$. Eyre et al., (2017) sequenced all GDH positive patients and compared the probability of faecal toxin positive and toxin negative patients being likely sources of transmission, that is, having $C$. difficile genetically linked to a subsequent isolate in another patient [30]. They concluded faecal toxin negative patients were similarly infectious to faecal toxin positive patients; faecal toxin status did not affect transmission rates [30]. They concluded strategies to identify and institute infection control measures around patients with potentially toxigenic $C$. difficile without detected faecal toxin are likely to reduce CDI incidence [30]. NAAT could be used as a screening test as it is rapid and highly sensitive to aid detection of such cases from an infection control perspective [31]. In addition with concerns about the lower sensitivity of toxin EIA testing increased usage of NAAT in hospitals worldwide has been seen [12]. The results in our study suggest a NAAT CT value of $\leq 26$ corresponds to toxin EIA positivity. In our setting, a GDH test followed by NAAT using the CT value as marker of EIA positivity would seem to be sufficient to pick up the majority of EIA positive cases. NAAT detects the DNA of the toxin gene of $C$. difficile rather than the presence of toxin in stool samples, however in our study a low CT value shows a strong association with toxin EIA positivity. Our finding is in line with the observations of Jazmati et al., (2016) that free toxin detection is associated with low CT-values [32]. As in our study Jazmati et al., (2016) showed CT-values were significantly lower in specimens that were positive in toxigenic culture $(26.2 \pm 4.5, n=100$ versus $30.5 \pm 5.0, n=$ 65; $p<0.001$ ) [32]. Senchyna et al., (2017) also found low $\mathrm{CT}$ values predicted free toxin results; a $\mathrm{CT}$ value of $<26.35$ could sensitively predict $96.0 \%$ of toxin EIA / PCR stool samples with a negative predictive value of $97.1 \%$ and with a specificity of $78.0 \%$ using both EIA toxin and cell cytotoxin assay [33]. In our study it is important to note that the toxin EIA has a sensitivity of $94.7 \%$ which could affect the CT value of $\leq 26$ corresponding to toxin EIA positivity. One main disadvantage of NAATs is that they do not detect the presence of biologically active toxin in stool specimens [15]. Toxins expressed by $C$. difficile are the main virulence factor, and some feel that the presence of toxin in stool is a positive correlation of disease $[4,12,15]$. The significance of detecting $C$. difficile in the absence of the toxins, such as in the patient who tests positive by NAAT but negative by toxin EIA, is unclear [15].

The majority of large studies on C. difficile diagnostics show toxin EIA is the best predictor of outcome, with NAAT tests providing no additional information about disease severity $[4,9,11,13]$. However, in our study $72 \%(n=99 / 138)$ patients with a CT value between 18 and 21 had severe/recurrent CDI based on PHE guidelines for management of CDI [20]. In the mild cases, with a CT value between 18 and 21, we often saw treatment failure with our first line therapy of metronidazole requiring escalation of treatment. In contrast, 92\% $(n=74 / 80)$ of the patients with a high $\mathrm{CT}$ value had mild CDI and responded to treatment. In our setting, the CT value could be used to guide treatment of patients with CDI. It must be noted we have only looked at a small number of patients with either the lowest or highest CT values. Reigadas et al., (2016) also showed patients with severe disease had significantly lower CT-values (toxin B CT value of $<23.5$ ) compared with patients in the other groups [12]. In our study, we also looked at the mortality within a month of CDI. It must be noted we have looked at all-cause mortality and not mortality specifically attributed to CDI. However, a significant association between low CT value and mortality was observed. Rao et al. (2015) found that there was no correlation between amplification CT values and severe CDI or all-cause mortality, however in our study; the mean toxin B amplification CT value was 27.1, which was much lower than that reported by Rao et al. (34.3) [34]. There were limitations in the study by Rao et al., (2015) which included sampling amounts and specimen transport. [34]

It is often difficult to distinguish between healthcare associated diarrhoea and CDI [1]. In CDI, the spectrum of clinical disease ranges from mild diarrhoea to toxic megacolon, colonic perforation and death [1]. Research suggests that both strain characteristics and the host's immune response influence CDI severity, recurrence risk and mortality [1]. Jazmati et al., Reigadas et al., and Senchyna et al., all showed a correlation between low NAAT CT values and severity and/or patient outcome $[12,32,33]$. To our knowledge, this is the largest study reporting a significant association between CT values of a NAAT $C$. difficile test vs toxin EIA positivity and mortality. There is growing evidence that NAAT CT values could be used to predict CDI severity, recurrence risk and mortality. 


\section{Conclusion}

Using ROC analysis to identify a relationship between CT value and EIA toxin positivity we were able to predict a cut off CT value threshold of $\leq 26$ which was associated with a high probability of EIA positivity. In addition, we showed a low CT value was associated with mortality within a month. Low CT values were also associated with more severe cases of CDI in our setting. A $C T$ value of $\leq 26$ could be used as an adjunct in the testing algorithm for $C$. difficile to enable detection of toxin EIA positive strains, as well as be used in conjunction with clinical parameters to assess disease severity and guide treatment of patients with CDI. However, we recognise that more work is needed to understand the nature of any link between low CT values and severity of CDI. We also suggest that, since a low CT value correlates with a positive toxin EIA, a CT $\leq 26$ could in theory be used to guide reporting and streamline the contentious testing issues of CDI.

Finally, we hypothesise that increased presence of $C$. difficile in the gastrointestinal tract of affected individuals leads to enhanced toxin detection, resulting in lower $\mathrm{CT}$ values and reflecting the phenomenon of gut dysbiosis that is thought to precipitate this disease.

\section{Acknowledgements \\ We would like thank the Infection Prevention and Control Team at the University Hospitals Birmingham NHS Foundation Trust for their help in the} generation of the data in this paper.

\section{Funding}

None.

Availability of data and materials

Not applicable.

\section{Authors' contributions}

All authors have contributed to the manuscript. Craig Bradley and Elisabeth Holden have helped draft the manuscript. Martyn Wilkinson has analysed all the data. The lead author Mark Garvey has written and prepared the manuscript.

\section{Ethics approval and consent to participate}

Not applicable.

\section{Consent for publication}

Not applicable.

\section{Competing interests}

The authors declare that they have no competing interests.

\section{Publisher's Note}

Springer Nature remains neutral with regard to jurisdictional claims in published maps and institutional affiliations.

Received: 29 September 2017 Accepted: 23 November 2017 Published online: 19 December 2017

\section{References}

1. Martin JS, Monaghan TM, Wilcox MH. Clostridium difficile infection: epidemiology, diagnosis and understanding transmission. Nat Rev Gastroenterol Hepatol. 2016;13:206-16.
2. Mawer DPC, Eyre DW, Griffiths D, et al. Contribution to Clostridium difficile transmission of symptomatic patients with toxigenic strains who are fecal toxin negative. Clin Infect Dis. 2017;64:1163-70.

3. Polage CR, Solnick JV, Cohen SH. Toxin immunoassays and Clostridium difficile infection-reply. JAMA Intern Med. 2016;176:414-5.

4. Planche TD, Davies KA, Coen PG, et al. Differences in outcome according to Clostridium difficile testing method: a prospective multicentre diagnostic validation study of C difficile infection. Lancet Infect Dis. 2013;13:936-45.

5. Klevens RM, Edwards JR, Richards CL Jr, et al. estimating health care associated infections and deaths in US hospitals, 2002. Public Health Rep. 2007;122:160-6.

6. Goldenberg SD, French GL. Diagnostic testing for Clostridium difficile: a comprehensive survey of laboratories in England. J Hosp Infect. 2011;79:4-7.

7. Wilcox MH. Laboratory diagnosis of Clostridium difficile infection: in a state of transition or confusion or both? J Hosp Infect. 2011:79:1-3.

8. Novak-Weekley SM, Marlowe EM, Miller JM, et al. Clostridium difficile testing in the clinical laboratory by use of multiple testing algorithms. J Clin Microbiol. 2010;48:889-93.

9. Planche T, Wilcox M. Reference assays for Clostridium difficile infection: one or two gold standards? J Clin Pathol. 2011;64:1-5.

10. Planche T, Aghaizu A, Holliman R, et al. Diagnosis of Clostridium difficile infection by toxin detection kits: a systematic review. Lancet Infect Dis. 2008:8:777-84.

11. Wilcox MH, Planche T, Fang FC, Gilligan P. Point-counterpoint. What is the current role of algorithmic approaches for diagnosis of Clostridium difficile infection? J Clin Microbiol. 2010;48:4347-53.

12. Reigadas E, Alcalá L, Valerio M, Marín M, Martin A, Bouza E, Toxin BPCR. Cycle threshold as a predictor of poor outcome of Clostridium difficile infection: a derivation and validation cohort study. J Antimicrob Chemother. 2016:71:1380-5.

13. Ticehurst JR, Aird DZ, Dam LM, et al. Effective detection of toxigenic Clostridium difficile by a two-step algorithm including tests for antigen and cytotoxin. J Clin Microbiol. 2006;44:1145-9.

14. Eastwood K, Else P, Charlett a, Wilcox M. Comparison of nine commercially available Clostridium difficile toxin detection assays, a real-time PCR assay for C. difficile $t c d B$, and a glutamate dehydrogenase detection assay to cytotoxin testing and cytotoxigenic culture methods. J Clin Microbiol. 2009; 47:3211-7.

15. Humphries RM, Uslan DZ, Rubin Z. Performance of Clostridium difficile toxin enzyme immunoassay and nucleic acid amplification tests stratified by patient disease severity. J Clin Microbiol. 2013;51:869-73.

16. Reller ME, Lema CA, Perl TM, et al. Yield of stool culture with isolate toxin testing versus a two-step algorithm including stool toxin testing for detection of toxigenic Clostridium difficile. J Clin Microbiol. 2007;45:3601-5.

17. Crobach MJ, Planche T, Eckert C, et al. European society of clinical microbiology and infectious diseases: update of the diagnostic guidance document for Clostridium difficile infection. Clin Microbiol Infect. 2016;22:S63-81

18. Cohen SH, Gerding DN, Johnson S, et al. Clinical practice guidelines for Clostridium difficile infection in adults: 2010 update by the society for healthcare epidemiology of America (SHEA) and the Infectious Diseases Society of America (IDSA). Infect Control Hosp Epidemiol. 2010;31:431-55.

19. Davies KA, Longshaw CM, Davis GL, et al. Under diagnosis of Clostridium difficile across Europe: the European, multicentre, prospective, biannual, point-prevalence study of Clostridium difficile infection in hospitalised patients with diarrhoea (EUCLID). Lancet Infect Dis. 2014;14:1208-19.

20. Updated guidance on the management and treatment of Clostridium difficile infection. 2013. Public Health England. https://www.gov.uk/ government/uploads/system/uploads/attachment_data/file/321891/ Clostridium_difficile_management_and_treatment.pdf.

21. SMI B10: Processing of faeces for Clostridium difficile, PHE, 2014 available at: https://www.gov.uk/government/publications/smi-b-10-processing-offaeces-for-clostridium-difficile.

22. Wren MW, Sivapalan M, Kinson R, Shetty NR. Laboratory diagnosis of Clostridium difficile infection. An evaluation of tests for faecal toxin, glutamate dehydrogenase, lactoferrin and toxigenic culture in the diagnostic laboratory. Br J Biomed Sci. 2009;66:1-5.

23. Lewis SJ, Heaton KW. Stool form scale as a useful guide to intestinal transit time. Scand J Gastroenterol. 1997;32:920-4.

24. hmeasure package. Version 1.0. R version 3.4 .0 (2017-04-21).

25. plotROC package. Version 2.0.1. R version 3.4 .0 (2017-04-21). 
26. R version 3.4.0 (2017-04-21) - "You Stupid Darkness". Copyright (C) 2017 The R Foundation for Statistical Computing.

27. Hand DJ. Measuring classifier performance: a coherent alternative to the area under the ROC curve. Mach Learn. 2009;77:103-23.

28. Lopez-Raton M, Rodriguez-Alvarez M, Cadarso-Suarez C, Gude-Sampedro F. OptimalCutpoints: an R package for selecting optimal Cutpoints in diagnostic tests. J Stat Soft. 2014;61:1-36.

29. Dionne LL, Raymond F, Corbeil J, Longtin J, Gervais P, Longtin Y. Correlation between Clostridium difficile bacterial load, commercial real-time PCR cycle thresholds, and results of diagnostic tests based on enzyme immunoassay and cell culture cytotoxicity assay. J Clin Microbiol. 2013;51:3624-30.

30. Eyre DW, Fawley WN, Rajgopal A, et al. Comparison of control of Clostridium Difficile infection in six English hospitals using whole-genome sequencing. Clin Infect Dis. 2017;65:433-41.

31. Mermel LA. Diverse sources of C. difficile infection. N Engl J Med. 2014:370: $182-3$.

32. Jazmati N, Hellmich M, Ličanin B, Plum G, Kaasch AJPCR. Cycle threshold value predicts the course of Clostridium difficile infection. Clin Microbiol Infect. 2016;22:e7-8.

33. Senchyna F, Gaur RL, Gombar S, Truong CY, Schroeder LF, Banael N. Clostridium Difficile PCR cycle threshold predicts free toxin. J Clin Microbiol. 2017:55:2651-60.

34. Rao K, Micic D, Natarajan M, et al. Clostridium difficile ribotype 027 relationship to age, detectability of toxins a or B in stool with rapid testing, severe infection, and mortality. Clin Infect Dis. 2015;61:233-41.

\section{Submit your next manuscript to BioMed Central} and we will help you at every step:

- We accept pre-submission inquiries

- Our selector tool helps you to find the most relevant journal

- We provide round the clock customer support

- Convenient online submission

- Thorough peer review

- Inclusion in PubMed and all major indexing services

- Maximum visibility for your research

Submit your manuscript at www.biomedcentral.com/submit 